\title{
Utility of glycosylated haemoglobin in diagnosing diabetes in an urban Sri Lankan community
}

\author{
C N Wijekoon ${ }^{1}$, A Pathmeswaran ${ }^{1}$, S Chackrewarthy ${ }^{1}$, N Kato ${ }^{2}$, A R Wickremasinghe ${ }^{1}$ \\ (Index words: HbA1c, fasting plasma glucose, diabetes, diagnose, Sri Lanka)
}

\begin{abstract}
Introduction American Diabetes Association (ADA) has officially endorsed glycosylated haemoglobin ( $\mathrm{HbA} 1 \mathrm{c})$ as a diagnostic tool. The recommended cut-off for diagnosing diabetes is $\geq 6.5 \%$.

Objectives To compare use of $\mathrm{HbA} 1 \mathrm{c}$ and fasting plasma glucose (FPG) to diagnose diabetes in an urban Sri Lankan community.

Methods This cross-sectional study is based on baseline data from a prospective study on non-communicable diseases in randomly selected individuals aged 35-64 years in a selected community. HbA1c was measured by National Glycohaemoglobin Standardization Program certified Bio Rad Variant HbA1c HPLC method. Diagnostic performance of $\mathrm{HbA1c}$ was evaluated in those without previous diabetes. Receiver Operating Characteristic Curve was used to identify optimum $\mathrm{HbA1c}$ threshold.
\end{abstract}

Results We studied 2516 individuals with no previous history of diabetes. Of these $53.8 \%$ were women. Mean age was $52 \pm 7.9$ years. FPG was $\geq 7 \mathrm{mmol} / /$ in 245 (9.7\%). HbA1c was $\geq 6.5 \%$ in 173 (6.9\%). Concordance between FPG and HbA1c was 95\% (both criteria positive: $5.8 \%$; both criteria negative: $89.2 \%$ ). Compared to FPG, HbA1c cut-off of $\geq 6.5 \%$ had specificity of $98.9 \%(95 \% \mathrm{Cl} 98.3-99.3)$ and sensitivity of $60 \%$ (95\% Cl 53.6-66.2). Positive and negative predictive values were $85 \%(95 \% \mathrm{Cl} 78.8-89.9)$ and $95.8 \%(95 \%$ Cl 94.9-96.6), respectively. Compared to FPG, optimum $\mathrm{HbA1c}$ threshold for diagnosing diabetes was $5.9 \%$ (sensitivity: 84\%; specificity: $88.8 \%$; area under the curve: 0.91).

Conclusions In the study population, detection of diabetes with ADA recommended $\mathrm{HbA1}$ c criterion was $29 \%$ less than with FPG criterion. Compared to FPG, HbA1c had high specificity but sensitivity was low. Further research is needed to refine the optimum $\mathrm{HbA1c}$ threshold in Sri Lankans.

Ceylon Medical Journal 2017; 62: 57-62

DOI: http://doi.org/10.4038/cmj.v62i1.8435

\section{Introduction}

Diabetes mellitus has become a global epidemic [1]. During the last decade it emerged as an important health concern in South Asian region [2]. In a nationally representative sample of Sri Lankan adults, prevalence of diabetes was reported to be $10.3 \%$ in 2006 and an increasing trend has been observed [2,3].

Macro and micro vascular complications of diabetes cause high morbidity and mortality globally. Early diagnosis would provide opportunities for prevention of these complications and would be cost effective for the individual as well as for the community [4].

There are several tools available for diagnosing diabetes. Fasting plasma glucose (FPG) measurement and the oral glucose tolerance test (OGTT) have been used for decades. Glycosylated haemoglobin (HbA1c) is a well-established measure of glycaemic control in those diagnosed with diabetes but until recent years it had not been considered suitable as a diagnostic tool due to lack of standardization of laboratory procedure. However, during the last decade many studies have suggested that HbA1c is a useful tool for diagnosing diabetes [5]. Furthermore, the measurement of $\mathrm{HbA}_{1 \mathrm{c}}$ has become more accurate and standardized in recent years [6]. In 2009, an International Expert Committee, with members appointed by the American Diabetes Association (ADA), the European Association for the Study of Diabetes (EASD) and the International Diabetes Federation (IDF), recommended the use of (HbA1c) to diagnose diabetes [7]. The following year ADA officially endorsed $\mathrm{HbA}_{1 \mathrm{c}}$ as a diagnostic test and recommended the cut-off value of $\geq 6.5 \%$ for diagnosing diabetes [8]. However, the ADA highlighted that the diagnostic test should be performed using a method that is certified by the National Glycohaemoglobin Standardization Program (NGSP) and standardized or traceable to the Diabetes Control and Complications Trial reference assay.

${ }^{1}$ Faculty of Medicine, University of Kelaniya, Ragama, Sri Lanka and ${ }^{2}$ International Medical Centre of Japan, Shinjuku-ku, Tokyo, Japan.

Correspondence: CNW, e-mail:<nirmalawijekoon@yahoo.co.uk>. Received 20 October 2016 and revised version accepted 23 January 2017. 
The main limitation in using HbA1c in resource poor settings is its cost. In Sri Lanka, the cost of HbA1c is about 5 times the cost of FPG and about 1.5 times the cost of OGTT. Nevertheless, HbA1c which is a marker of chronic glycaemia has several advantages over FPG and OGTT which are markers of acute glycaemia. Being markers of acute glycaemia both FPG and OGTT can show intra-individual variability due to factors like diet, exercise, stress and illness. Such variability is minimal with HbA1c. The HbA1c assay does not require a fasting or timed sample and thus is more convenient for the individual. There is evidence to suggest greater preanalytical stability of HbA1c over FPG and OGTT [9]. Furthermore, OGTT is less acceptable to individuals due to the cumbersome procedure and it is more labour and time consuming. There is evidence that $\mathrm{HbA} 1 \mathrm{c}$ has a more consistent relationship with diabetic retinopathy than does FPG [10].

However, there is controversy regarding applicability of ADA recommended HbA1c cut-off in different ethnic groups. According to studies from different countries, suggested cut-off to diagnose diabetes ranged from $\geq 5.6 \%$ to $\geq 7.0 \%$ [11-24]. Age, ethnicity, genetic makeup and erythrocyte life-span are likely to contribute to inter-individual variability of glycosylation of haemoglobin and could be responsible for observed differences in the cut-offs in different populations [15, 19, 25-27].

With the increasing prevalence of non-communicable diseases in Sri Lanka, there is a need to screen the community for diabetes. Even though OGTT is considered as the gold standard in diagnosing diabetes, in actual clinical practice it is not often utilized except during pregnancy. The most widely used diagnostic tool in clinical practice in Sri Lanka is FPG. However, access to laboratories with standardized protocols for HbA1c testing is increasing and the use of HbA1c for diagnosis of diabetes has become popular especially in the private sector. The performance of HbA1c, in comparison to the other diagnostic tools has been widely studied in many countries. However, there is only limited data available regarding its diagnostic utility among Sri Lankans. This study aims to explore the utility of HbA1c in diagnosing diabetes in Sri Lankan adults compared to FPG which is the most widely used diagnostic tool in Sri Lanka.

\section{Methods}

This study is based on the analysis of the baseline data from a large prospective study on non-communicable diseases in a selected community [Ragama Health Study (RHS)] conducted by the Faculty of Medicine, University of Kelaniya, Sri Lanka in collaboration with the National Center for Global Health and Medicine, Tokyo, Japan. Baseline data collection was carried out from January to September 2007. Detailed methodology of RHS has been previously described [28]. Study methods in brief are given below.

\section{Study setting and participants}

RHS was conducted in the Ragama Medical Officer of Health $(\mathrm{MOH})$ area (area $=25 \mathrm{~km}^{2}$, Grama Niladhari divisions $=21$, housing units $=15,137$, population at the time of study=75,591). Adults aged between 35-64 years were randomly selected using the electoral register of each Grama Niladhari division as the sampling frame. The selected individuals were visited at their homes and invited to participate in the study.

\section{Measurements and assays}

The selected participants attended a special screening clinic set up at the Faculty of Medicine, University of Kelaniya. The participants were given specific written and verbal instructions to attend this clinic after an overnight fast of 12 hours. Demographic, anthropometric and clinical data were recorded by trained investigators. Ten milliliters of venous blood was drawn for assay of HbA1c, FPG and lipid profile. The collected blood samples were centrifuged and separated immediately. FPG was measured according to the hexokinase method. HbA1c was measured by National Glycohaemoglobin Standardization Program certified ion exchange HPLC (Variant, Bio-Rad, USA) method.

\section{Ethical aspects}

Approval was obtained from the Ethical Review Committee of Faculty of Medicine, University of Kelaniya, Sri Lanka. Participants were recruited to the study after obtaining informed written consent. A special follow-up clinic was commenced at the Faculty of Medicine, University of Kelaniya to provide treatment and follow-up to those found to have abnormal findings.

\section{Definitions}

Self-reported medical history and information from medical records such as diagnosis cards and clinic record books was utilized to document presence of previously diagnosed diabetes. In those without previous diabetes, ADA recommended criteria (FPG $\geq 7 \mathrm{mmol} / \mathrm{l}$ and $\mathrm{HbA} 1 \mathrm{c}$ $\geq 6.5 \%$ ) were utilized to identify newly detected diabetes [8].

\section{Statistical analysis}

Data were entered into a database developed in Epi Info (Centers for Disease Control and Prevention, Atlanta, GA, USA) and logical and range checks were done. Statistical analysis was done using Stata version 8 (Stata Corp, College Station, Texas 77845, USA). Continuous variables were summarized using means and standard deviations. Categorical variables were summarized as percentages. Individuals without previous diabetes, were classified into four mutually exclusive groups based on the ADA recommended FPG and HbA1c 
criteria for diabetes; participants meeting both (FPG $\geq 7$ $\mathrm{mmol} / \mathrm{l}$ and $\mathrm{HbA} 1 \mathrm{c} \geq 6.5 \%$ ), neither (FPG $<7 \mathrm{mmol} / \mathrm{l}$ and $\mathrm{HbA} 1 \mathrm{c}<6.5 \%$ ), and only one of the criteria (FPG $\geq 7$ $\mathrm{mmol} / \mathrm{l}$ and $\mathrm{HbA} 1 \mathrm{c}<6.5 \%$ or FPG $<7 \mathrm{mmol} / \mathrm{l}$ and $\mathrm{HbA} 1 \mathrm{c}$ $\geq 6.5 \%$ ). Sensitivity, specificity, positive predictive value and negative predictive value were calculated for HbA1c as compared to FPG. Receiver Operating Characteristic Curve (ROC) was used to identify the optimum $\mathrm{HbA}_{1 \mathrm{c}}$ threshold. FPG value of $\geq 7 \mathrm{mmol}$ was considered as diagnostic of diabetes and that was used as the gold standard in undertaking ROC analysis to identify the ideal cut-off value of HbA1c to diagnose diabetes mellitus.

\section{Results}

Out of the 4200 randomly selected individuals, 3012 participated in the Ragama Health Study (response rate of $72 \%$ ) and complete data were available for 2985 participants. Four hundred and sixty nine (15.7\%) had previously diagnosed diabetes.

Performance of HbA1c criterion as a diagnostic tool for diabetes was evaluated in 2516 participants without previous diabetes. Among them 1353 (53.8\%) were women and the mean age was $52(\mathrm{SD}=7.9)$ years. The characteristics of these individuals are described in table 1.

Table 1. Demographic, anthropometric and clinical characteristics of subjects without previously diagnosed diabetes $(n=2516)$

\begin{tabular}{|c|c|c|c|}
\hline \multirow[b]{2}{*}{ Characteristic } & \multicolumn{3}{|c|}{ Mean $[S D]$} \\
\hline & $\begin{array}{c}\text { All } \\
(n=2516)\end{array}$ & $\begin{array}{c}\text { Men } \\
(n=1163)\end{array}$ & $\begin{array}{c}\text { Women } \\
(n=1353)\end{array}$ \\
\hline FPG (mmol/l) & $6.0[1.5]$ & $6.0[1.4]$ & $6.0[1.6]$ \\
\hline HbA1c (\%) & $5.6[1.0]$ & $5.5[0.9]$ & $5.6[1.0]$ \\
\hline $\begin{array}{l}\text { Systolic blood pressure } \\
\text { (mmHg) }\end{array}$ & $133.9[21.7]$ & $133.0[20.7]$ & $134.7[22.5]$ \\
\hline $\begin{array}{l}\text { Diastolic blood pressure } \\
\text { (mmHg) }\end{array}$ & $79.2[12.4]$ & $79.1[12.7]$ & $79.4[12.2]$ \\
\hline Body Mass Index (kg/m²) & $24.0[4.3]$ & $23.0[4.0]$ & $24.9[4.5]$ \\
\hline Total cholesterol (mmol/l) & $5.5[1.1]$ & $5.3[1.1]$ & $5.6[1.1]$ \\
\hline Triglyceride (mmol/l) & $1.4[0.8]$ & $1.5[0.9]$ & $1.3[0.7]$ \\
\hline
\end{tabular}

FPG - Fasting Plasma Glucose; HbA1c - Glycosylated Haemoglobin

Among those without previous diabetes, FPG was $\geq 7 \mathrm{mmol} / \mathrm{l}$ in 245 (9.7\%) and HbA1c was $\geq 6.5 \%$ in 173 (6.9\%). Hence detection rate of diabetes with HbA1c criterion was 29\% less than with FPG criterion. Both
HbA1c and FPG criteria for diabetes were positive in 147 (5.8\%) and both were negative in 2245 (89.2\%) thus the concordance between FPG and HbA1c was 95\%. Ninety eight (3.9\%) individuals who were positive for FPG criterion were negative for HbA1c criterion whereas 26 (1\%) individuals who were positive for HbA1c criterion were negative for FPG criterion (Table 2). Compared to FPG criterion, ADA recommended HbA1c criterion had a sensitivity of 60\% (95\% CI 53.6-66.2) and specificity of $98.9 \%$ (95\% CI 98.3-99.3). The positive and negative predictive values were $85 \%$ (95\% CI 78.8-89.9) and 95.8\% (95\% CI 94.9-96.6), respectively.

Table 2. Number of subjects with newly detected diabetes based on ADA recommended HbA1c criterion and FPG criterion $(n=2516)$

\begin{tabular}{lccc}
\hline & \multicolumn{3}{c}{ HbA1c } \\
\cline { 2 - 3 } FPG & $\begin{array}{l}<6.5 \% \\
(n, \%)\end{array}$ & $\begin{array}{l}\geq 6.5 \% \\
(n, \%)\end{array}$ & $\begin{array}{l}\text { Total } \\
(n, \%)\end{array}$ \\
\hline$<7 \mathrm{mmol} / \mathrm{l}$ & $2245(89.2)$ & $26(1)$ & $2271(90.2)$ \\
$\geq 7 \mathrm{mmol} / \mathrm{l}$ & $98(3.9)$ & $147(5.8)$ & $245(9.7)$ \\
Total & $2343(93.1)$ & $173(6.9)$ & $2516(100)$ \\
\hline
\end{tabular}

FPG - Fasting Plasma Glucose; HbA1c - Glycosylated Haemoglobin

Figure 1 shows the ROC curve (area under curve 0.91) for HbA1c using FPG $\geq 7 \mathrm{mmol} / \mathrm{l}$ as the gold standard. Compared to FPG, the optimum HbA1c threshold for detecting diabetes in the study population was $5.9 \%$. This threshold has a sensitivity of $84 \%$, specificity of $88.8 \%$ (Table 3 ).

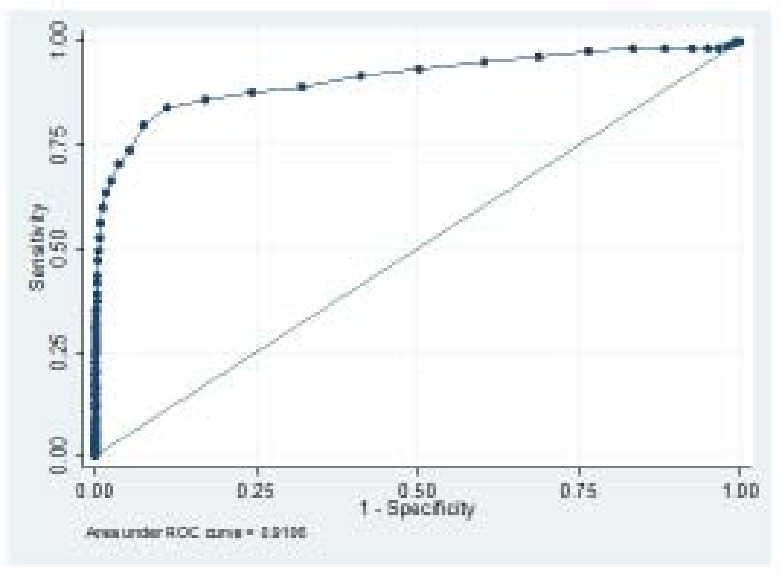

Figure 1. Receiver operating characteristic curve for detecting diabetes using FPG and $\mathrm{HbA1c}$ criteria 


\section{Table 3. Sensitivity and specificity of different HbA1c cut-off compared to FPG criterion (FPG $\geq 7 \mathrm{mmol} / \mathrm{l}$ )}

\begin{tabular}{lcc}
\hline HbA1C cut-off & Sensitivity $\%$ & Specificity $\%$ \\
\hline$\geq 4.9 \%$ & 98.4 & 11.6 \\
$\geq 5.4 \%$ & 93.4 & 49.8 \\
$\geq 5.9 \%$ & $\mathbf{8 4 . 0}$ & $\mathbf{8 8 . 8}$ \\
$\geq 6.2 \%$ & 70.5 & 96.3 \\
$\geq 6.5 \%$ & 59.9 & 98.9 \\
$\geq 7.0 \%$ & 43.4 & 99.7 \\
$\geq 8.0 \%$ & 27.1 & 99.9 \\
$\geq 8.3 \%$ & 25.0 & 100 \\
\hline
\end{tabular}

\section{Discussion}

Even though HbA1c obtained endorsement as a diagnostic tool for diabetes more than 5 years ago, data regarding its diagnostic utility among Sri Lankans is scarce. Our study provides valuable evidence comparing HbA1c with FPG in diagnosing diabetes in Sri Lankan adults. FPG is the most widely used diagnostic tool in Sri Lanka. The main strength of our study is its randomly selected community based large sample. This study is based on the analysis of the baseline data from a prospective study on non-communicable diseases in randomly selected individuals in a selected community. The response rate for baseline data collection was high and rate of missing information was low. Though a formal sample size calculation was not done for the purpose of assessing the validity of $\mathrm{HbA} 1 \mathrm{c}$, the narrow $95 \%$ confidence intervals for the sensitivity, specificity and the positive and negative predictive values indicate that the sample size was adequate.

In the study population, prevalence of previously undetected diabetes was high. Among those with no previous history of diabetes, $5.8 \%$ fulfilled both $\mathrm{HbA1c}$ and FPG criteria recommended by ADA for diagnosing diabetes. 9.7\% had FPG above the ADA recommended cut-off while only $6.9 \%$ had HbA1c above the ADA cutoff. Accordingly, in this population the HbA1c criterion of $\geq 6.5 \%$ identified $29 \%$ fewer cases of diabetes than the FPG criterion of $\geq 7 \mathrm{mmol} / \mathrm{l}$. Analysis of National Health and Nutrition Examination Survey (NHANES) data indicated that the HbA1c cut-off of $\geq 6.5 \%$ identifies one-third fewer cases of undiagnosed diabetes than the FPG cut-off of $\geq 7 \mathrm{mmol} / \mathrm{l}$ [8]. In the new Hoorn study, Van't Riet et al showed that when using OGTT as the gold standard for diagnosing diabetes, FPG had a higher area under the ROC curve than HbA1c [12]. This indicates that FPG is better than HbA1c in detecting previously undiagnosed diabetes. In our study population, when both positive and negative results were considered the two tests were in agreement with each other in $95 \%$ of the cases (concordance rate was 95\%). When compared with the FPG criterion, HbA1c cut off of $\geq 6.5 \%$ had a high specificity but the sensitivity was low. These findings are comparable with previous studies from different parts of the world [13, 14, 17-19, 23].

A certain degree of discrepancy between the FPG and HbA1c criteria was anticipated from the outset. Which one does better remains an unresolved issue. When these two are compared there is no doubt that HbA1c is more convenient and theoretically it should be more accurate as a diagnostic tool because it is a marker of chronic glycaemia whereas FPG and OGTT are markers of acute glycaemia which are liable to intraindividual variability due to several factors. Even though some evidence indicate that $\mathrm{HbA} 1 \mathrm{c}$ is a better predictor of vascular complications of diabetes, long term follow up studies are needed to clarify the issue regarding the most accurate diagnostic tool [10]. These studies would be difficult to conduct and such evidence may not be available for many years.

As compared with the FPG criterion, the optimum HbA1c threshold for detecting diabetes in this Sri Lankan population was $5.9 \%$ which is lower than the ADA cutoff. This is consistent with most of the studies from other countries. Almost similar thresholds have been reported in Korea and India [16, 17]. There are several other studies from China, USA, Bulgaria, Bangladesh, Malaysia, and in the Middle East which found that the optimum HbA1c thresholds were lower than the ADA cut-off [14,19-22,29,30]. However, one study of the indigenous population in Australia reported an optimum cut-off of 7\% [23]. A systematic review of nine studies done in Europe and Asia reported that the optimum HbA1c cut-off was 6.1\% [5]. A recently published study done in 254 Sri Lankans using OGTT as the reference, has reported an optimum HbA1c cut-off of 6.3\% [31]. All these results including the result from our study highlight that for better utilization of $\mathrm{HbA1c}$ as a diagnostic tool it needs to be further refined to suit different populations.

\section{Limitations}

There are several limitations to our study. The reference investigation we used to assess the performance of HbA1c in diagnosing diabetes was FPG and not the OGTT which is considered as the gold standard. Therefore, we may have missed a group of people with normal FPG but elevated 2-hour plasma glucose. The actual optimum HbA1c cut-off may have been different if the diagnosis was based on OGTT instead of FPG. Nevertheless, our findings are consistent with reports that the optimum HbA1c cut-off is lower than the ADA recommended cut-off. The aim of the study was to explore the performance of HbA1c as compared to the widely used FPG and we believe that the other findings, such as the rate of detection of diabetes with ADA recommended HbA1c cut-off compared to FPG, concordance between the two investigations and the sensitivity, specificity and 
positive and negative predictive values of $\mathrm{HbA} 1 \mathrm{c}$ compared to FPG, are all useful information for individual clinicians as well as policy makers. Confounders such as severe iron deficiency anaemia and haemoglobinopathies which could alter the HbA1c value have not been excluded. Finally, this study was conducted in a single urban community and these results may not be generalisable to the entire country.

\section{Conclusions}

Our findings add evidence to the limited evidence base regarding the utility of HbA1c as a diagnostic tool for diabetes in Sri Lankans and it contributes to the ongoing discussions worldwide regarding the potential value and ideal cut-off of HbA1c in the diagnosis of diabetes. In this urban Sri Lankan adult population, ADA recommended $\mathrm{HbA} 1 \mathrm{c}$ criterion identified one-third fewer cases of undiagnosed diabetes than the FPG criterion. Compared to FPG, HbA1c had high specificity but the sensitivity was low which is similar to data from several studies from different countries. It is important to consider these facts when HbA1c and FPG reports are interpreted for the purpose of diagnosing diabetes. Nationally-representative data from large scale studies and long term follow up cohort studies are required to refine the optimum HbA1c threshold for Sri Lankans.

\section{Acknowledgement}

This work was supported by a grant from the National Center for Global Health and Medicine, Tokyo, Japan.

\section{Conflicts of interest}

There are no conflicts of interest.

\section{References}

1. International Diabetes Federation. Diabetes Atlas. 7th ed. Brussels, Belgium: International Diabetes Federation, 2015. Available at http://www.diabetesatlas.org/ (Accessed on Oct 07, 2016)

2. Jayawardena R, Ranasinghe P, Byrne NM, Soares MJ, Katulanda P, Hills AP. Prevalence and trends of the diabetes epidemic in South Asia: a systematic review and metaanalysis. BMC Public Health 2012; 12: 380.

3. Katulanda P, Constantine GR, Mahesh JG, et al. Prevalence and projections of diabetes and pre-diabetes in adults in Sri Lanka - Sri Lanka Diabetes, Cardiovascular Study (SLDCS). Diabet Med 2008; 25: 1062-9.

4. Gillies CL, Abrams KR, Lambert PC, et al. Different strategies for screening and prevention of type 2 diabetes in adults: cost-effectiveness analysis. BMJ 2008; 336: 1180-5.
5. Bennett CM, Guo M, Dharmage SC. HbA1c as a screening tool for detection of type 2 diabetes: a systematic review. Diabet Med 2007; 24: 333-43.

6. Hanas R, John G. International HbA1c Consensus Committee. 2010 Consensus Statement on the Worldwide Standardization of the Hemoglobin A1c Measurement. Clin. Chem. 2010; 56: $1362-4$.

7. The International Expert Committee. International Expert Committee report on the role of the A1C assay in the diagnosis of diabetes. Diabetes Care 2009; 32: 1327-34.

8. American Diabetes Association. Diagnosis and Classification of Diabetes Mellitus. Diabetes Care 2010; 33: S62-9.

9. Little RR, Rohlfing CL, Tennill AL, Connolly S, Hanson S. Effects of sample storage conditions on glycated hemoglobin measurement: evaluation of five different high performance liquid chromatography methods. Diabetes Technol Ther 2007; 9: 36-42.

10. Tapp RJ, Tikellis G, Wong TY, Harper CA, Zimmet PZ, Shaw JE. Longitudinal association of glucose metabolism with retinopathy. Diabetes Care 2008; 31: 1349-54.

11. Hu Y, Liu W, Chen Y, et al. Combined use of fasting plasma glucose and glycated hemoglobin A1c in the screening of diabetes and impaired glucose tolerance. Acta Diabetol 2010; 47: 231-6.

12. van’t Riet E, Alssema M, Rijkelijkhuizen JM, Kostense PJ, Nijpels G, Dekker JM. Relationship between A1C and glucose levels in the general Dutch population: the new Hoorn study. Diabetes Care 2010; 33: 61-6.

13. Rohlfing CL, Little RR, Wiedmeyer HM, et al. Use of GHb (HbA1c) in screening for undiagnosed diabetes in the U.S. population. Diabetes Care 2000; 23: 187-91.

14. Bao Y, Ma X, Li H, et al. Glycated haemoglobin A1c for diagnosing diabetes in Chinese population: cross sectional epidemiological survey. BMJ 2010; 340: c2249.

15. Davidson MB, Schriger DL. Effect of age and race/ethnicity on HbA1c levels in people without known diabetes mellitus: implications for the diagnosis of diabetes. Diabetes Res Clin Pract 2010; 87: 415-21.

16. Kim JH, Kim GW, Lee MY, et al. Role of HbA1c in the Screening of Diabetes Mellitus in a Korean Rural Community. Diabetes Metab J 2012; 36: 37-42.

17. Nair M, Prabhakaran D, Venkat Narayan KM, et al. HbA1c values for defining diabetes and impaired fasting glucose in Asian Indians. Prim Care Diabetes 2011; 5: 95-102.

18. Huffman FG, Exebio JC, Zarini GG, Exebio C. Use of HbA (1c) in screening for Cuban-Americans with undiagnosed type 2 diabetes. J Immigr Minor Health 2011; 13: 541-5.

19. Lipska KJ, De Rekeneire N, Van Ness PH, et al. Identifying dysglycemic states in older adults: implications of the emerging use of hemoglobin A1c. J Clin Endocrinol Metab 2010; 95: 5289-95. 
20. Tankova T, Chakarova N, Dakovska L, Atanassova I. Assessment of HbA1c as a diagnostic tool in diabetes and prediabetes. Acta Diabetol 2012; 49: 371-8.

21. Bhowmik B, Diep LM, Munir SB, et al. HbA1c as a diagnostic tool for diabetes and pre-diabetes: the Bangladesh experience. Diabet Med 2013; 30: e70-e77.

22. Wan Nazaimoon WM, Md Isa SH, Wan Mohamad WB, et al. Prevalence of diabetes in Malaysia and usefulness of HbA1c as a diagnostic criterion. Diabet Med 2013; 30: 825-8.

23. Rowley KG, Daniel M, O’Dea K. Screening for diabetes in Indigenous populations using glycated haemoglobin: sensitivity, specificity, post-test likelihood and risk of disease. Diabet Med 2005; 22: 833-9.

24. Mohan V, Vijayachandrika V, Gokulakrishnan K, et al. HbA1c cut points to define various glucose intolerance groups in Asian Indians. Diabetes Care 2010; 33: 515-9.

25. Soranzo N, Sanna S, Wheeler E, et al. Common variants at 10 genomic loci influence hemoglobin A1C levels via glycemic and nonglycemic pathways. Diabetes 2010; 59: 3229-39.

26. Jørgensen ME, Bjerregaard P, Borch-Johnsen K, Witte D.
New diagnostic criteria for diabetes: is the change from glucose to HbA1c possible in all populations? J Clin Endocrinol Metab 2010; 95: E333-6.

27. Kilpatrick ES, Bloomgarden ZT , Zimmet PZ . Is haemoglobin A1c a step forward for diagnosing diabetes? BMJ 2009; 339: b4432.

28. Dassanayake AS, Kasturiratne A, Rajindrajith S, et al. Prevalence and risk factors for non-alcoholic fatty liver disease among adults in an urban Sri Lankan population. $J$ Gastroenterol Hepatol 2009; 24: 1284-8.

29. Pinelli NR, Jantz AS, Martin ET, Jaber LA. Sensitivity and specificity of glycated hemoglobin as a diagnostic test for diabetes and prediabetes in Arabs. $J$ Clin Endocrinol Metab 2011; 96: E1680-3.

30. Kharroubi AT, Darwish HM, Abu Al-Halaweh AI, Khammash UM. Evaluation of Glycated Hemoglobin (HbA1c) for Diagnosing Type 2 Diabetes and Prediabetes among Palestinian Arab Population. PLoS One 2014; 9: e88123.

31. Herath HM, Weerarathna TP, Dahanayake MU, Weerasinghe NP. Use of HbA1c to diagnose type 2 diabetes mellitus among high risk Sri Lankan adults. Diabetes Metab Syndr. 2016; pii: S1871-4021(16)30175-8. 